\title{
ESTIMATION OF ETONOGESTREL IN HUMAN PLASMA BY USING LC-ESI-MS/MS METHOD
}

\author{
SATISH RAMANATHAM VELAMAKANNI ${ }^{* 1,2}$, VENKATESWARLU PADALA ${ }^{2}$
}

${ }^{1}$ Bioanalytical Lab, Vimta Labs Limited, Cherlapally, Hyderabad 500051, India, 2University College of Chemistry, Jawaharlal Nehru Technological University, Kukatpally, Hyderabad 500085, India

Email: velamakanni4273@gmail.com

Received: 25 Jun 2017 Revised and Accepted: 02 Nov 2017

\section{ABSTRACT}

Objective: The aspiration of the present study was to develop simple, robust and reliable liquid chromatography/electrospray ionization tandem mass spectrometry (LC-MS/MS) (Agilent Technologies) assay method for the quantification of etonogestrel in human Plasma by using etonogestrel $\mathrm{d}_{6}$ as internal standard (IS).

Methods: An easy Liquid-Liquid Extraction (LLE) sample processing method was used to extract etonogestrel from plasma and chromatographic method was developed with run time $3.5 \mathrm{~min}$ with linear calibration curve ranges from $50-3604 \mathrm{pg} / \mathrm{ml}$ for both etonogestrel and etonogestrel $\mathrm{d}_{6}$ and chromatographic method validated by determining carryover test, sensitivity, matrix effect, linearity, precision, accuracy, recovery, dilution integrity and stability. The developed method was used for the pharmacokinetic study of 75 mcg desogestrel tablet formulation under fasting condition in healthy females.

Results: The validation showed that the developed method was accurate with the results of validated parameters were met acceptance criteria as per Food and Drug Administration (FDA) guidelines. The validated method successfully was used for the pharmacokinetic study of 75mcg desogestrel tablet in healthy females and quantified the amount of etonogestrel and IS.

Conclusion: The developed method for etonogestrel in human plasma has been validated and used in pharmacokinetic studies.

Keywords: Etonogestrel, Liquid-Liquid Extraction, Human plasma, Pharmacokinetics

(C) 2017 The Authors. Published by Innovare Academic Sciences Pvt Ltd. This is an open-access article under the CC BY license (http://creativecommons.org/licenses/by/4.0/) DOI: http://dx.doi.org/10.22159/ijpps.2017v9i12.20946

\section{INTRODUCTION}

Etonogestrel or 3-ketodesogestrel is a steroidal progestin used in hormonal contraceptives which prevents pregnancy (release of an egg from an ovary) associated with changes in cervical mucus and uterine lining [1] (fig. 1). Implants such as nexplanon and implanon and nuva ring are the only brands used for the birth control. The pill which is used to control the birth rate instead of the implants is Cerazette [2]. Depending on the rate and magnitude of the increase observed in plasma, etonogestrel is given orally with a single oral dose of the two tablets of $75 \mathrm{mcg}$ tablets to adults. Under fasting or fed conditions etonogestrel is well tolerated at all doses and can be administered and its absolute bioavailability of etonogestrel $85 \%$ is reported [3]. According to the literature, different methods have been reported for the quantification of etonogestrel and most of them were not specified for etonogestrel [3-9].

The researchers Timmer et al. and Thomas et al. reported the quantification of etonogestrel in human serum samples with Limit of quantification (LOQ) $32.5 \mathrm{pg} / \mathrm{ml}$. The proposed methods were enough and sensitive for determination of etonogestrel, but there were some disadvantages like getting serum is a time-consuming process and at the time of serum collection blood has to be clotted and lot of time is wasted for centrifugation and low yields with a high risk of hemolysis and thrombocytolysis [10]. The reported methods have the more run time of chromatography and in sample processing used more volume of plasma consumption, and used Solid phase extraction which is more costly and also some were reported the methods non-deuterated internal standards (IS) $[8,11]$. In this point of view, the present study was carried out to develop new LC-MS method to prevail over the problem in the earlier developed methods [3-9] like matrix effect, more runtime, costeffective etc., for quantification of etonogestrel using deuterated etonogestrel (etonogestrel $\mathrm{d}_{6}$ ) by applying liquid-liquid extraction (LLE) extraction and we successfully developed the new method and validated it with sensitivity of $50.0 \mathrm{pg} / \mathrm{ml}$ using a low plasma volume $(50 \mu \mathrm{l})$. This sensitive method was successfully applied to a clinical pharmacokinetic study on healthy female subjects following the oral administration of desogestrel as described under fasting condition. The authenticity in the measurement of study data is demonstrated through incurred samples reanalysis.

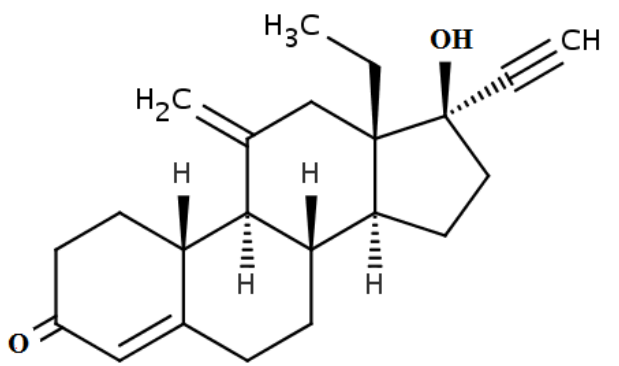

Fig. 1: Chemical structures of etonogestrel

\section{MATERIALS AND METHODS}

\section{Chemicals}

Etonogestrel (99.77\%) and etonogestrel $d_{6}$ (99.95\%) were obtained from Clearsynth Labs Limited (Mumbai, India), The control $\mathrm{K}_{2}-$ human plasma sample was procured from Cauvery Pathological Lab's (Hyderabad, India) and the other chemicals used in methods were analytical grade and HPLC grade. Reciprocating shaker from Scigenics Biotech, Chennai, India and Megafuse 3SR from Heraeus, Germany.

\section{LC-MS/MS instrument and conditions}

An HPLC system (Agilent Technologies, USA) consisting of a Zorbax SB-Phenyl column (100 mm × $4.6 \mathrm{~mm}, 3.5 \mu \mathrm{m}$; Agilent Technologies, Santa Clara, CA, USA), a binary LC-1200 G1312A prominence pump, an autosampler (LC-1200 G1367B) and a 
solvent degasser (LC-1200) was used for the study. Aliquot of $20 \mu \mathrm{l}$ of the processed samples were injected into the column, which was kept at $40{ }^{\circ} \mathrm{C}$. An isocratic mobile phase consisting of a mixture of methanol and $0.1 \%$ formic acid $(80: 20, \mathrm{v} / \mathrm{v})$ was used to separate the analyte from the endogenous components and delivered at a flow rate of $1.0 \mathrm{ml} / \mathrm{min}$ into the electrospray ionization chamber of the mass spectrometer. Quantification was achieved with MS-MS detection in positive ion mode for the analyte and the IS using an MDS Sciex API-4000 mass spectrometers (Foster City, CA, USA) equipped with a Turboionspray ${ }^{\mathrm{TM}}$ interface at $500{ }^{\circ} \mathrm{C}$. The ion spray voltage was set at $5500 \mathrm{~V}$. The source parameters viz. the nebulizer gas (GS1), auxiliary gas (GS2), curtain gas and collision gas were set at $40,40,15$, and 6 psi, respectively. The compound parameters viz. the declustering potential (DP), collision energy (CE), entrance potential (EP) and collision cell exit potential (CXP) were 72, 45, 15, $6 \mathrm{~V}$ for etonorgestrel and 75, 45, 10, $6 \mathrm{~V}$ for the IS. Detection of the ions was carried out in the multiple-reaction monitoring mode (MRM), by monitoring the transition pairs of $m / z 340.30$ precursor ion to the $\mathrm{m} / \mathrm{z} 124.20$ for etonogestrel and $\mathrm{m} / \mathrm{z} 346.30$ precursor ion to the $m / z 130.20$ production for the IS. Quadrupoles Q1 and Q3 were set to low and unit resolution. The analysis data obtained were processed by Analyst Software ${ }^{\mathrm{TM}}$ (version 1.4.2).

\section{Preparation of plasma standards and quality controls}

A standard stock solution of etonogestrel and IS $(1 \mathrm{mg} / \mathrm{ml})$ were prepared in methanol. Working solutions for calibration and controls were prepared by appropriate dilution in methanol and water (90:10, v/v; diluent). The IS working solution $(50 \mathrm{ng} / \mathrm{ml})$ was prepared by diluting its stock solution with diluent. Stock solutions of etonogestrel and IS were found to be stable for more than $23 \mathrm{~h}$ at room temperature.

Calibration samples were prepared by spiking $950 \mu \mathrm{l}$ of control $\mathrm{K}_{2}$ EDTA human plasma with the $50 \mu \mathrm{l}$ working standard solution of the analyte as a bulk, to obtain etonogestrel concentration levels of 50 , $100,250,500,750,1200,2402$ and $3604 \mathrm{pg} / \mathrm{ml}$ as a single batch at each concentration. Similarly, quality control (QC) samples were also prepared as a bulk based on an independent weighing of standard drug, at concentrations of 50 (LLOQ), 150.00 (low), 525 (Geometric mean), 1800 (middle 1) and $3003 \mathrm{pg} / \mathrm{ml}$ (high) as a single batch at each concentration. The calibration and control bulk samples were divided into aliquots in microcentrifuge tubes (Tarson, $2 \mathrm{ml}$ ) and stored in the freezer at $-70 \pm 10^{\circ} \mathrm{C}$ until analyses.

\section{Sample processing}

All frozen subject samples, calibration standards and quality control samples were thawed and allowed to room temperature prior before analysis. The samples were vortexed for 10 seconds to mix prior to spiking. A $50 \mu \mathrm{l}$ aliquot of human plasma sample was mixed with $20 \mu \mathrm{L}$ of the IS working solution $\left(50 \mathrm{ng} / \mathrm{ml}\right.$ of etonogestrel $\left.\mathrm{d}_{6}\right)$. After vortex for 10 seconds, a $2.5 \mathrm{ml}$ of extraction solvent (ethyl acetate: cyclohexane (1:1)) was added using dispenser. The sample was shaken for $20 \mathrm{~min}$ using a reciprocating shaker and then centrifuged for $10 \mathrm{~min}$ at $4000 \mathrm{rpm}$ on Megafuse 3SR. The clear organic layer $(2 \mathrm{ml})$ was transferred to a $2 \mathrm{ml}$ glass test tube and evaporated at $45{ }^{\circ} \mathrm{C}$ under a gentle stream of nitrogen. The dried extract was reconstituted with $200 \mu \mathrm{l}$ of the mobile phase followed by the treatment with $30 \mu \mathrm{l}$ of hydroxylammonium chloride(1M) solution and kept in the incubator at $60{ }^{\circ} \mathrm{C}$ for 10 min which undergoes derivatization, showed in below equation. A $20 \mu \mathrm{l}$ aliquot of it was injected into the LC-MS/MS system.

$$
\mathrm{C}_{22} \mathrm{H}_{28} \mathrm{O}_{2}+\mathrm{NH}_{2} \mathrm{OH} \rightarrow \mathrm{C}_{22} \mathrm{H}_{29} \mathrm{O}_{2} \mathrm{~N}+\mathrm{H}_{2} \mathrm{O}
$$

\section{Bioanalytical method validation}

A systematic and complete method validation of a developed method for quantification of etonogestrel in human plasma was carried out as per US FDA guidelines [12]. The parameters determined were carryover test, selectivity, specificity, sensitivity, matrix effect, linearity, precision, accuracy, recovery, dilution integrity and stability. Carryover experiment was performed to verify any carryover of the analyte and IS, which may reflect in subsequent runs. The design of the study comprised of the following sequence of injections i.e. blank plasma sample $\rightarrow$ six samples of
LLOQ $\rightarrow$ blank plasma sample $\rightarrow$ ULOQ sample $\rightarrow$ blank plasma samples to check for any interference due to carry over. Selectivity was assessed by comparing the chromatograms of six different batches of blank plasma obtained from six different sources including one lipemic and hemolyzed plasma. Potential interference from the commonly used drugs, acetaminophen, nicotine, ibuprofen, caffeine, aspirin, and theophylline were evaluated. Sensitivity was determined by analyzing six replicates of plasma samples spiked with the lowest level of the calibration curve concentrations. Matrix effect was checked with six different lots of $\mathrm{K}_{2}$ EDTA plasma. Three replicate samples each of LQC and HQC were prepared from different lots of plasma (36 QC samples in total). The linearity of the method was determined by analysis of standard plots associated with a eight-point (non-zero standards) standard calibration curve. In addition, blank plasma samples were also analyzed to confirm the absence of direct interferences. To determine intra-day accuracy and precision, a calibration curve and six replicates of LLOQ QC, LQC, GMQC, MQC and HQC were analyzed on the same day. Inter-day accuracy and precision were assessed by analyzing three batches of samples on two consecutive days. Recoveries of analyte and IS were determined by comparing the peak area of extracted analyte standard with the peak area of non-extracted standard. Recovery of etonogestrel was determined at a concentration of 150 (low), 1800 (middle) and 3003 (high) pg/ml, whereas for IS was determined at concentration of $50 \mathrm{ng} / \mathrm{ml}$. Dilution integrity was performed to extend the upper concentration limit with acceptable precision and accuracy. Six replicates each at a concentration of about 1.50 times of the uppermost calibration standard were diluted one-and four-fold with blank plasma. The diluted samples were processed and analyzed.

Stability tests were conducted to evaluate the analyte stability in stock solutions and in plasma samples under different conditions. The stock solution stability at room temperature and refrigerated conditions $\left(2-8{ }^{\circ} \mathrm{C}\right)$ was performed by comparing the area response of the analyte (stability samples) with the response of the sample prepared from fresh stock solution. Bench top stability $(24 \mathrm{~h})$, processed samples stability (autosampler stability for $34 \mathrm{~h}$, wet extract stability for $28 \mathrm{~h}$ and dry extract stability for $28 \mathrm{~h}$ ), freezethaw stability (3 cycles), long-term stability to be performed at LQC and HQC levels using six replicates at each level. Samples were considered to be stable if assay values were within the acceptable limits of accuracy $( \pm 15 \% \mathrm{SD})$ and precision $(\leq 15 \% \mathrm{RSD})$.

\section{Pharmacokinetic study design}

A single dose pharmacokinetic study was performed in healthy female subjects $(n=6)$. The Ethics Committee (Samkshema Independent Ethics Committee, Hyderabad, India) approved the protocol and the volunteers provided with written informed consent. The subjects were fasted $10 \mathrm{~h}$ before administration of the drug formulation. Six healthy South Indian female subjects with an age group of 19-36 y and body-mass index (BMI) of $\geq 18.5 \mathrm{~kg} / \mathrm{m}^{2}$ and $\leq 30.0 \mathrm{~kg} / \mathrm{m}^{2}$, with body weight not less than $45 \mathrm{~kg}$ were chosen for the study. They were given a single oral dose $(2 \times 75 \mathrm{mcg})$ of desogestrel tablets. Blood samples were collected at pre-dose and $0.33,0.67,1,1.25,1.5,1.75,2,2.25,2.5,2.75,3,3.5,4,6.00,8,12,24,36$, 48, 60 and $72 \mathrm{~h}$, in $\mathrm{K}_{2}$ EDTA vacutainer ( $5 \mathrm{ml}$ ) collection tubes (BD, Franklin, NJ, USA). The tubes were centrifuged at $4500 \mathrm{rpm}$ for $10 \mathrm{~min}$ and the plasma was collected. The collected plasma samples were stored at $-70 \pm 10^{\circ} \mathrm{C}$ till their use. Plasma samples were spiked with the IS and processed as per the extraction procedure described earlier.

The main pharmacokinetic parameters of the etonogestrel were calculated by the non-compartmental model using WinNonlin Version 5.2. An incurred sample re-analysis was conducted by selecting the 24 subject samples $(2$ samples from each period of each subject) near $C_{\max }$ and the elimination phase. The percent change in the value should not be more than $\pm 20 \%$ [13].

\section{RESULTS AND DISCUSSION}

\section{Mass spectrometry}

Mass parameters were tuned in positive and negative ionization modes using electrospray ionization source for the analyte and the IS at a concentration of $100 \mathrm{ng} / \mathrm{ml}$ tuning solution during method development. The response observed was much higher in positive 
ionization mode compared to the negative mode for the analyte and IS may be due to their basic nature and use of small amount of formic acid $(0.1 \%)$ in the mobile phase further enhanced the detection of analyte and IS with low background noise, resulting in higher sensitivity. Protonated form of the analyte and IS, $[\mathrm{M}+\mathrm{H}]+$ ion was the parent ion in the $Q_{1}$ spectrum and was used as the precursor ion to obtain $Q_{3}$ product ion spectra. The most sensitive mass transition was observed from m/z 340.3 to 124.2 for etonogestrel and from m/z 346.3 to 130.2 for the IS (fig. 2, fig. 3). The dwell time for each transition was $200 \mathrm{~ms}$.

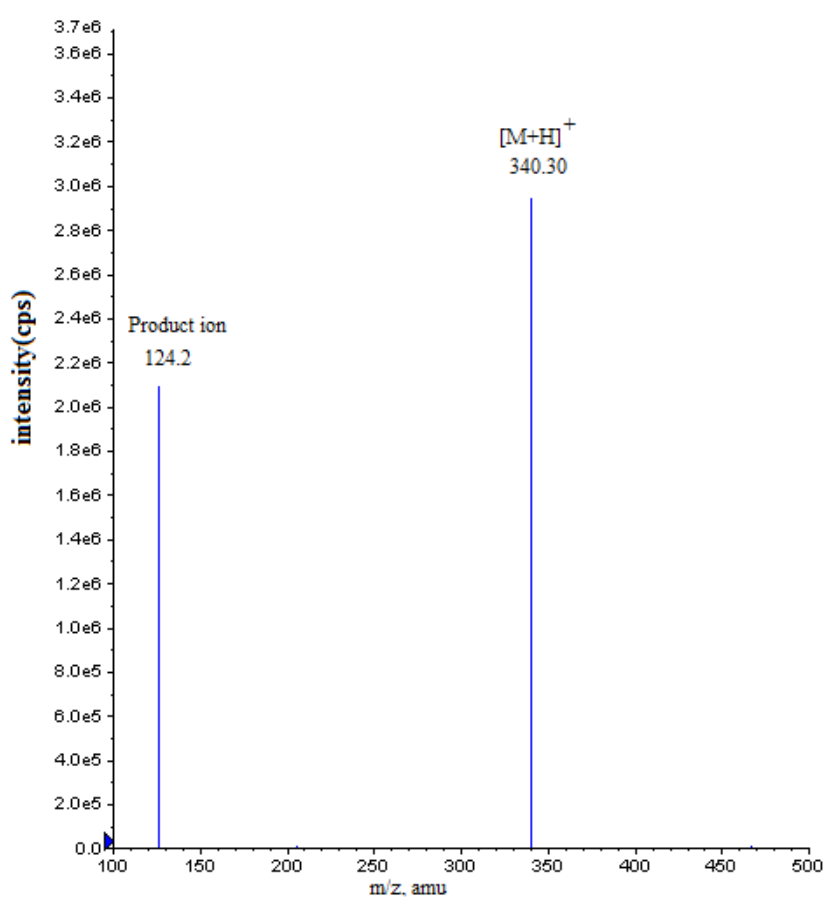

Fig. 2: Product ion mass spectra of $[\mathrm{M}+\mathrm{H}]+$ of etonogestrel

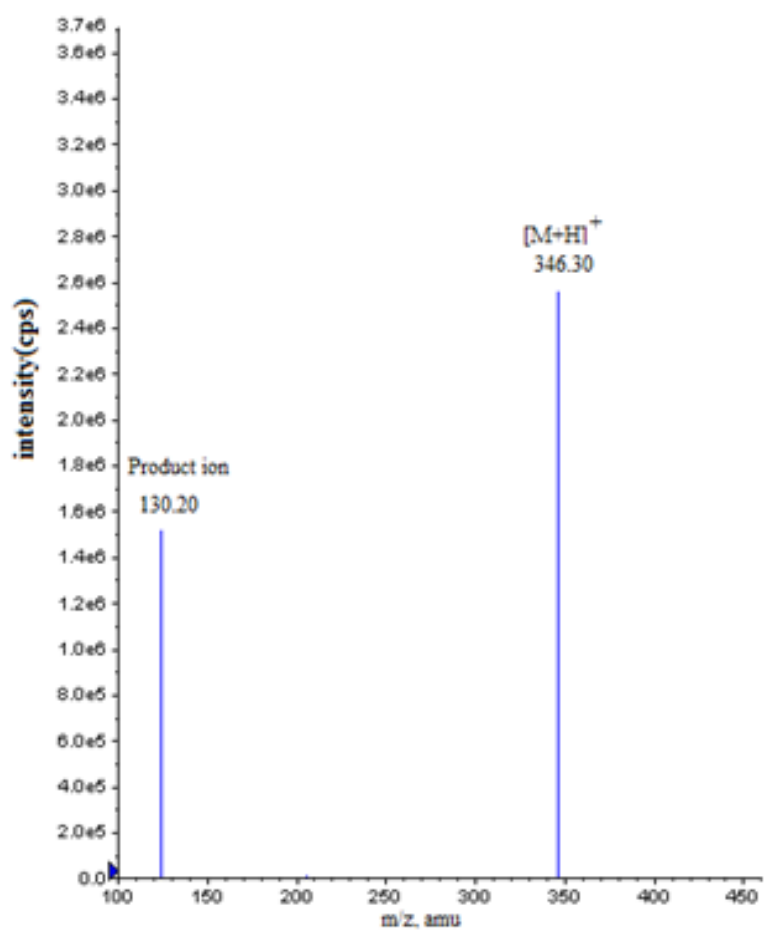

Fig. 3: Product ion mass spectra of $[M+H]+o f$ etonogestrel $d_{6}$ (IS)

\section{Method development}

The quantification of different analytes at different levels in biological matrices was a distinctive process using LC-MS at bioanalytical laboratories [14-19]. To develop a sensitive and selective bio-analytical method requires the judicious selection of chromatography column, mobile phases [20]. These parameters should be carefully monitored to produce the required resolution from endogenous components which in turn affect sensitivity and reproducibility of the analytical method by ion suppression. Once chromatographic column, mobile phase $\mathrm{pH}$ and organic solvent are set then flow rate, column temperature and buffer type and concentration can be managed for optimal response. As the objective of the present study, a fully validate an LC-MS/MS method for the determination of etonogestrel in human plasma with high sensitivity was developed as below.

Separation was attempted using organic solvents such as acetonitrile and methanol in different volume ratio with buffers like ammonium formate, ammonium acetate $(2-20 \mathrm{mmol})$ as well as acid additives like acetic acid and formic acid (0.1-0.5\%) in varying strength on different columns like $\mathrm{C}_{8}, \mathrm{C}_{18}$ and Phenyl of different makes (Hypurity advance, Zorbax, Inertsil, Ace, Grace, Kromasil, Hypersil etc).

Finally, methanol and $0.1 \%$ formic acid $(80: 20, v / v)$ as the mobile phase was most appropriate to give best sensitivity, efficiency and peak shape in Zorbax SB-Phenyl (100 x $4.6 \mathrm{~mm}, 3.5 \mu \mathrm{m})$ column even at low concentrations $(50 \mathrm{pg} / \mathrm{ml})$ of analyte. In addition, the effect of flow rate was also studied from 0.3 to $1.0 \mathrm{ml} / \mathrm{min}$, which was also responsible for acceptable chromatographic peak shapes and finally flow rate was set at $1.0 \mathrm{ml} / \mathrm{min}$. The retention time of analyte and the IS were low enough (2.2 min for both) allowing a chromatographic run time of $3.5 \mathrm{~min}$.

The method specified by Thomas et al. (2013) reported LLE extraction procedure for the extraction of etonogestrel and this includes usage of the solvent dichloromethane by employing progesterone $d_{8}$ as IS and serum was used as matrix. Extraction of etonogestrel was done employing SPE specifies usage of the high volume of $0.7 \mathrm{ml}$ plasma [8]. But, the method reported was may not be suitable because of the long run time and inconsistent results by the use of the non-deuterated standard as IS and high amount of plasma usage was done which is not suitable for commercial purposes to do more samples in stipulated time and also costeffective. Based on different types of reported methods, we tried diverse types of solvents were tried as single and in combination such as diethyl ether, cyclohexane, tert-butyl methyl lether, hexane, ethyl acetate, heptane and dichloromethane. Among these trails ethyl acetate: cyclohexane (1:1) was found to be optimal, which can produce a clean chromatogram for a blank sample and yields the highest recovery for the analyte from the plasma of analyte. There was no significant improvement in the recovery of the analyte by addition of acidic or basic additives to the plasma samples in different volume ratios. By the above, finally, LLE was employed for this work and obtained high recoveries of about $98 \%$ at all QC levels. Thus, in the present work by employing LLE lot of time is saved and its very simple method [15].

\section{Carryover effect}

Carryover was done to confirm that it does not affect the precision and accuracy of the specified method. After injection of the highest concentration of the analyte (ULOQ; the upper limit of quantitation), there was no significant carryover observed in a blank sample which specifies no carryover of the analyte in subsequent samples.

\section{Selectivity and chromatography}

By analyzing blank human plasma extract [fig. 4 (A)] and an extract spiked only with the IS [fig. 4 (B)] the selectivity of the method was confirmed. In drug-free human plasma at the retention time of the analyte and IS as shown in fig. 4 (A), no significant interference in blank plasma was observed from endogenous substances. Likewise, from the IS to the MRM channel of the analyte, which shows the absence of direct interference. Fig. 4 (C) indicates a representative ion-chromatogram for the LLOQ (STD-A) sample $(50.0 \mathrm{pg} / \mathrm{ml})$. Use 
of common medications such as acetaminophen, nicotine, acetylsalicylic acid, ibuprofen, caffeine and theophylline does not show interference. Representative chromatograms resulting from the analysis of subject blank plasma sample shown in the fig. 5 (A) and $1.25 \mathrm{~h}$ subject plasma sample after the administration of a $75 \mathrm{mcg}$ oral double dose of desogestrel is shown in the fig. 5 (B).

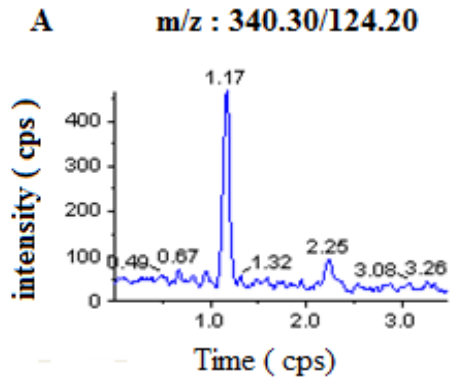

B

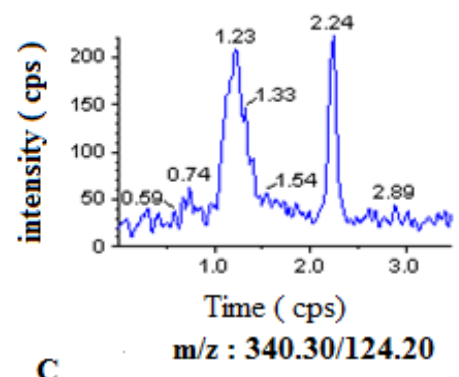

C

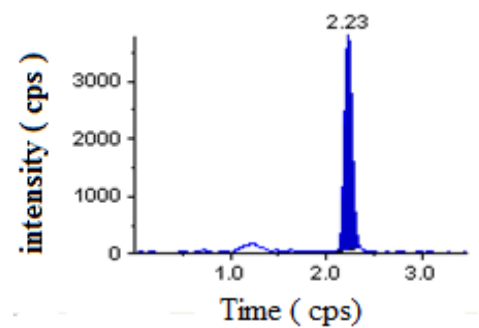

$\mathrm{m} / \mathrm{z}: 346.30 / 130.20$

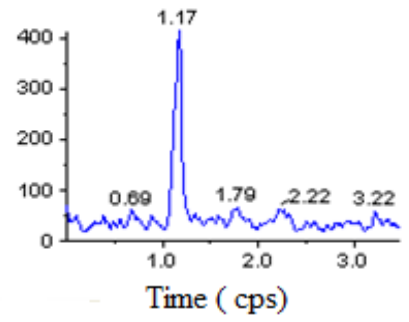

$\mathrm{m} / \mathrm{z}: \mathbf{3 4 6 . 3 0 / 1 3 0 . 2 0}$
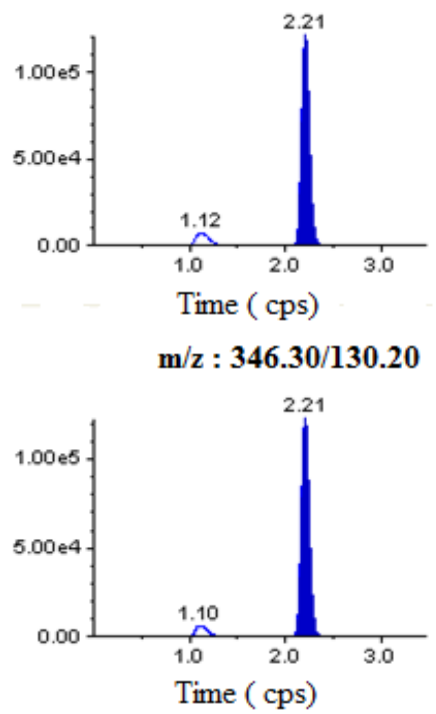

Fig. 4: Typical MRM chromatograms of etonogestrel (Left Panel) and IS (Right Panel) in human blank plasma (A), and human plasma spiked with IS (B), A LLOQ sample along with IS (C)

\section{Matrix effect}

Matrix effect assessment was done with the aim to check the effect of different lots of plasma on the back calculated value of $\mathrm{QC}$ 's nominal concentration. The results found were well within the acceptable limits (table 1). No significant matrix effect was observed in all the six batches of human plasma for the analyte at low and high-quality control concentrations. Also, the extraction method was rugged enough and gave accurate and consistent results when applied to real subject samples.

\section{Linearity, precision and accuracy}

Over the concentration range of $50.0-3604 \mathrm{pg} / \mathrm{ml}$ for etonogestrel, the eight-point calibration curve was found to be linear. To produce the best fit for the concentration-detector response relationship the two weighting models $\left(1 / x\right.$ and $\left.1 / x^{2}\right)$ were compared, a regression equation with a weighting factor of $1 / \mathrm{x}^{2}$ of the drug to the IS concentration was found. The mean correlation coefficient of the weighted calibration curves generated during the validation was $\geq 0.99$.

The intraday and inter-day precision deviation values were all within $15 \%$ of the relative standard deviation (RSD) at the low, middle and high-quality control level, whereas within $20 \%$ at LLOQ QCs level (table 2). The intraday and inter-day accuracy deviation values were all within $100 \pm 15 \%$ of the actual values at low, middle, and high-quality control level, whereas within $100 \pm 20 \%$ at LLOQ QCs level. The results show good precision and accuracy.

\section{Recovery}

Six replicates at low, medium-2 and high-quality control concentration for etonogestrel were prepared for recovery determination. The mean overall recovery of etonogestrel was $98.8 \%$ with the precision range of $0.75-4.31 \%$ and the recovery of IS was $99.2 \%$ with the precision of $1.58-2.10 \%$. The recoveries of analyte and IS were good and reproducible.

Thus, for the bioanalysis, the assay has been proved to be robust.

\section{Stability studies and dilution integrity}

In the different stability experiments carried out viz. bench top stability $(24 \mathrm{~h})$, autosampler stability $(34 \mathrm{~h})$, wet extract stability $(28 \mathrm{~h})$, repeated freeze-thaw cycles (3 cycles), reinjection stability $(4 \mathrm{~h})$ and long-term stability to be proved the mean $\%$ nominal values of the analyte were found to be within $\pm 15 \%$ of the predicted concentrations for the analyte at their LQC and HQC levels (table 3).

Thus, the results were found to be within the acceptable limits during the entire validation.

Stock solutions of etonogestrel and IS were found to be stable for 23 $\mathrm{h}$ at room temperature. The percentage stability (with the precision range) of etonogestrel and IS was $103.02 \%(2.23-6.02 \%)$ for LQC $99.60 \%(0.47-1.50 \%)$ for HQC and $100.28 \%(2.38-2.46 \%)$ respectively. 
A

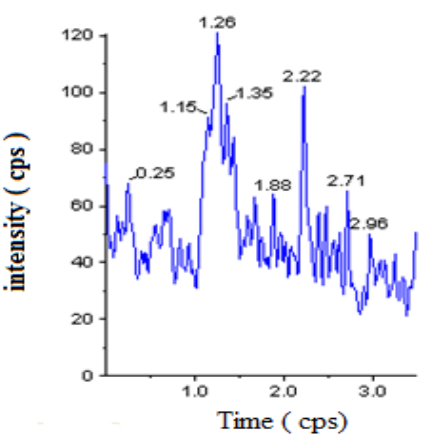

B

$\mathrm{m} / \mathbf{z}: \mathbf{3 4 0 . 3 0} / \mathbf{1 2 4 . 2 0}$

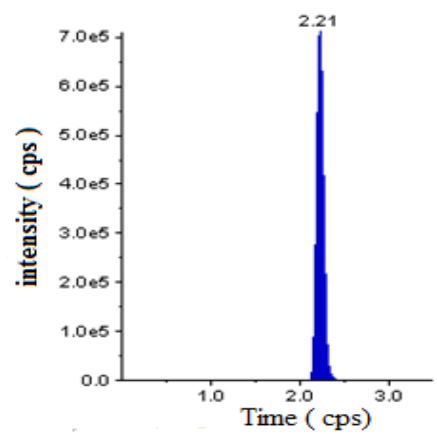

$\mathbf{m} / \mathbf{z}: \mathbf{3 4 6 . 3 0 / 1 3 0 . 2 0}$

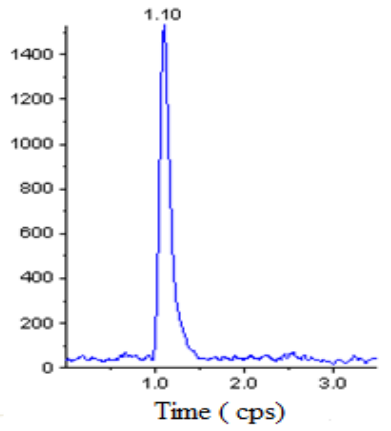

$\mathrm{m} / \mathrm{z}: \mathbf{3 4 6 . 3 0} / \mathbf{1 3 0 . 2 0}$

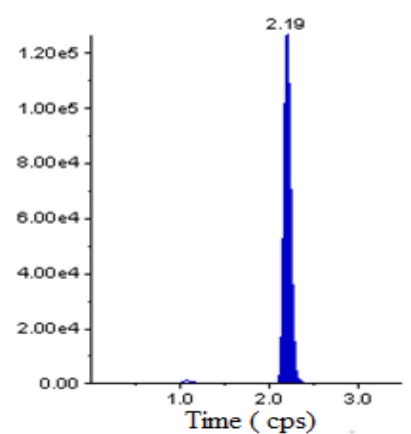

Fig. 5: MRM Chromatograms resulting from the analysis of subject blank plasma sample (A) and $1.25 \mathrm{~h}$ subject plasma sample (B), after the administration of a two tablets of $75 \mathrm{mcg}$ oral single dose of desogestrel tablet. The sample concentration was determined to be 1040 $\mathrm{pg} / \mathrm{ml}$

Table 1: Matrix effect of etonogestrel in human plasma $(N=6)$

\begin{tabular}{lllll}
\hline Plasma lot & LQC $(\mathbf{5 0 . 0} \mathbf{~ p g} / \mathbf{m l})$ & & HQC $(\mathbf{3 0 0 3} \mathbf{~ p g} / \mathbf{m l})$ & \\
\cline { 2 - 5 } & Concentration found $($ mean \pm SD; $\mathbf{~ g / m l )}$ & \% accuracy & Concentration found (mean \pm SD; ng/ml) & \% accuracy \\
\hline Lot 1 & $155 \pm 2.65$ & 103 & $3024 \pm 12.1$ & 101 \\
Lot 2 & $156 \pm 1.64$ & 104 & $3011 \pm 9.77$ & 100 \\
Lot 3 & $163 \pm 4.17$ & 108 & $3017 \pm 23.9$ & 100 \\
Lot 4 & $157 \pm 0.35$ & 104 & $3019 \pm 27.4$ & 101 \\
Lot 5 & $162 \pm 5.51$ & 108 & $3020 \pm 25.4$ & 101 \\
Lot 6 & $156 \pm 4.74$ & 103 & $3004 \pm 0.27$ & 100 \\
\hline
\end{tabular}

Where $(\mathrm{N}=6 \pm \mathrm{SD})$

Table 2: Precision and accuracy data for etonogestrel

\begin{tabular}{|c|c|c|c|c|}
\hline Quality control & Run & Concentration found (mean \pm Standard deviation, $\mathrm{pg} / \mathrm{ml}$ ) & Precision (\%) & Accuracy (\%) \\
\hline \multicolumn{5}{|c|}{ Intra-day variations (Six replicates at each concentration) } \\
\hline \multirow[t]{3}{*}{ LLOQ } & 1 & $49.7 \pm 4.84$ & 9.72 & 99.5 \\
\hline & 2 & $51.1 \pm 2.08$ & 4.08 & 102 \\
\hline & 3 & $53.6 \pm 4.04$ & 7.52 & 107 \\
\hline \multirow[t]{3}{*}{ LQC } & 1 & $147 \pm 9.92$ & 6.75 & 98 \\
\hline & 2 & $146 \pm 11.1$ & 7.61 & 97.5 \\
\hline & 3 & $153 \pm 6.44$ & 4.22 & 102 \\
\hline \multirow[t]{3}{*}{ MQC } & 1 & $1825 \pm 60.6$ & 3.32 & 101 \\
\hline & 2 & $1798 \pm 83.8$ & 4.66 & 99.9 \\
\hline & 3 & $1789 \pm 52.6$ & 2.94 & 99.4 \\
\hline \multirow[t]{3}{*}{$\mathrm{HQC}$} & 1 & $3101 \pm 55.8$ & 1.80 & 103 \\
\hline & 2 & $3093 \pm 54.4$ & 1.76 & 103 \\
\hline & 3 & $3088 \pm 69.8$ & 2.26 & 103 \\
\hline \multicolumn{5}{|c|}{ Inter-day variations (Eighteen replicates at each concentration) } \\
\hline LLOQ & & $51.5 \pm 3.96$ & 7.69 & 103 \\
\hline LQC & & $149 \pm 9.30$ & 6.25 & 99.1 \\
\hline MQC & & $1804 \pm 64.9$ & 3.60 & 100 \\
\hline HQC & & $3094 \pm 57$ & 1.84 & 103 \\
\hline
\end{tabular}


Table 3: Stability data for etonogestrel in plasma

\begin{tabular}{lllll}
\hline Stability test & QC (spiked concentration (pg/ml) & Mean \pm SD (pg/ml) & Precision (\%) & Accuracy/stability (\%) \\
\hline Process $^{\mathrm{a}}$ & 150 & $142 \pm 3.84$ & 2.70 & 94.6 \\
& 3003 & $3114 \pm 23.1$ & 0.74 & 104 \\
Process $^{\mathrm{b}}$ & 150 & $143 \pm 2.43$ & 1.70 & 95.2 \\
& 3003 & $3090 \pm 15.4$ & 0.50 & 103 \\
Bench top $^{\mathrm{c}}$ & 150 & $146 \pm 2.40$ & 1.65 & 97.1 \\
& 3003 & $3096 \pm 20.9$ & 0.68 & 103 \\
FT $^{\mathrm{d}}$ & 150 & $144 \pm 3.67$ & 2.55 & 96 \\
& 3003 & $3102 \pm 31.2$ & 1.01 & 103 \\
Reinjection $^{\mathrm{e}}$ & 150 & $150 \pm 7.92$ & 5.26 & 100 \\
& 3003 & $2951 \pm 174$ & 5.90 & 98.3 \\
\hline
\end{tabular}

aafter $34 \mathrm{~h}$ in an auto sampler at $20^{\circ} \mathrm{C}$, bafter $28 \mathrm{~h}$ of wet extract, cafter $24 \mathrm{~h}$ at room temperature, dafter 3 freeze and thaw cycles, eafter $4 \mathrm{~h}$ of Reinjection. Where $(\mathrm{N}=6 \pm \mathrm{SD})$

The upper concentration limit of etonogestrel can be extended to $5251 \mathrm{pg} / \mathrm{ml}$ for by $1 / 4$ dilutions with screened human blank plasma. The mean back-calculated concentrations for $1 / 4$ dilution samples were within $85-115 \%$ of their nominal value and the coefficients of variations $(\% \mathrm{CV})$ were less than $2.19 \%$.

\section{Pharmacokinetic study results}

The validated method was successfully applied for a pharmacokinetic study of etonogestrel in 6 healthy adult female subjects who received a double dose of $75 \mathrm{mcg}$ of desogestrel, respectively under fasting condition.

With the reported lowest LLOQ $(50.0 \mathrm{pg} / \mathrm{ml})$ etonogestrel was quantifiable beyond $72 \mathrm{~h}$ post-dosing (fig. 6 and table 4). The results of the present study were similar to the profile has been reported previously, for the $75 \mathrm{mcg}$ formulation [8].

\section{Incurred sample reanalysis}

At the Crystal City III meeting, the FDA has specified the importance and need of doing incurred sample reanalysis evaluation [12, 21]. By using dosed subject samples it is must to demonstrate assay reproducibility. Incurred sample reanalysis was performed by picking up of two plasma samples from each subject and re-assayed in a separate batch run.

The differences in concentrations between the ISR and the initial values for all the tested samples should be less than $20 \%$, indicating good reproducibility of the present method (table 5).

Table 4: Pharmacokinetic parameters for etonogestrel in healthy female subjects

\begin{tabular}{ll}
\hline PK parameter & Mean (SD) \\
\cline { 2 - 2 }$t_{\max }(\mathrm{h})$ & $\mathbf{1 5 0 \text { mg }}$ \\
$C_{\max }(\mathrm{pg} / \mathrm{ml})$ & $1.67 \pm 1.16$ \\
$\mathrm{AUC}_{0-\mathrm{t}}(\mathrm{pg} \mathrm{h} / \mathrm{ml})$ & $1031 \pm 70.3$ \\
$\mathrm{AUC}_{0-\infty}(\mathrm{pg} \mathrm{h} / \mathrm{ml})$ & $10781 \pm 3276$ \\
$\operatorname{Kel}^{-1}\left(\mathrm{~h}^{-1}\right)$ & $16574 \pm 5490$ \\
$\mathrm{t}_{1 / 2}(\mathrm{~h})$ & $0.02 \pm 0.03$ \\
\hline
\end{tabular}

Where $(\mathrm{N}=6 \pm \mathrm{SD})$

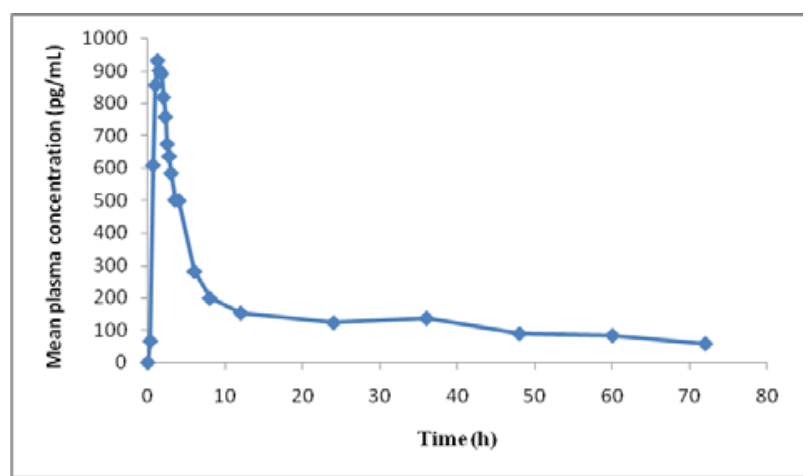

Fig. 6: Mean plasma concentration-time profile of etonogestrel in human plasma following oral administration of etonogestrel (two tablets of 75 mcg tablet) to healthy volunteers $(n=6)$

This paper demonstrates the successful development and validation of a simple, sensitive and rapid LC-MS/MS method for the determination of etonogestrel in human plasma samples according to commonly acceptable FDA guidelines [21]. The method presented has the highest sensitivity $(50.0 \mathrm{pg} / \mathrm{ml})$ and employs low plasma volume $(50 \mu \mathrm{l})$ for processing compared to other procedures, therefore the volume of the sample to be collected per time point from an individual during the study is reduced significantly-this allows inclusion of additional points. Moreover, the total analysis time (extraction and chromatography) is the shortest.
Thus, the advantage of this method is that a relatively more number of samples can be analyzed in short time, thus increasing the output. The simple LLE procedure with ethyl acetate: cyclohexane (1:1) minimizes the chances of errors, saves considerable time and simplifies the sample preparation procedure.

From the results of all the validation parameters, we can conclude that the developed method can be useful for bioavailability and bioequivalence (BA/BE) studies and routine therapeutic drug monitoring with the desired precision and accuracy. 
Table 5: Incurred samples reanalysis data of etonogestrel

\begin{tabular}{|c|c|c|c|}
\hline \multicolumn{4}{|c|}{ Desogestrel double dose of $75 \mathrm{mcg}$} \\
\hline Sample & Initial conc. $(\mathrm{pg} / \mathrm{ml})$ & Re-assay conc. $(\mathrm{pg} / \mathrm{ml})$ & Difference (\%) \\
\hline 1 & 891.062 & 870.143 & -2.38 \\
\hline 2 & 181.415 & 176.998 & -2.46 \\
\hline 3 & 755.365 & 760.409 & 0.67 \\
\hline 4 & 196.232 & 188.091 & -4.24 \\
\hline 5 & 930.126 & 933.091 & 0.32 \\
\hline 6 & 202.937 & 208.726 & 2.81 \\
\hline 7 & 964.971 & 971.908 & 0.72 \\
\hline 8 & 164.789 & 156.092 & -5.42 \\
\hline 9 & 974.615 & 980.043 & 0.56 \\
\hline 10 & 183.431 & 172.098 & -6.38 \\
\hline 11 & 926.301 & 951.052 & 2.64 \\
\hline 12 & 162.500 & 166.075 & 2.18 \\
\hline 13 & 906.305 & 917.802 & 1.26 \\
\hline 14 & 177.367 & 186.308 & 4.92 \\
\hline 15 & 766.332 & 733.269 & -4.41 \\
\hline 16 & 189.034 & 192.627 & 1.88 \\
\hline 17 & 924.501 & 941.206 & 1.79 \\
\hline 18 & 197.392 & 202.246 & 2.43 \\
\hline 19 & 933.278 & 948.679 & 1.64 \\
\hline 20 & 171.254 & 177.349 & 3.50 \\
\hline 21 & 956.856 & 968.403 & 1.20 \\
\hline 22 & 192.499 & 188.452 & -2.12 \\
\hline 23 & 933.596 & 943.445 & 1.05 \\
\hline 24 & 166.337 & 173.566 & 4.25 \\
\hline
\end{tabular}

\section{CONCLUSION}

The present work carried out to develop a new analytical method for quantification of etonogestrel in human plasma using LC-MS/MS and successfully developed the analytical method and proved to be specific, selective, linear, precise, accurate, robust, fast, and reliable. The developed method successfully applied to quantify the etonogestrel in the healthy female subjects.

\section{ACKNOWLEDGEMENT}

The authors gratefully acknowledge Vimta Labs Limited (Hyderabad, India) for providing necessary facilities to carry out this work.

\section{AUTHOR CONTRIBUTION}

Satish Ramanatham Velamakanni has collected the data, designed the study, developed the methodology and performed the anlaysis, as his $\mathrm{PhD}$ Thesis. Venkateswarlu Padala contributed to data analysis and interpretation.

\section{CONFLICT OF INTERESTS}

We have none to declare

\section{REFERENCES}

1. Milsom I, T Korver. Ovulation incidence with oral contraceptives: a literature review. J Fam Plann Reprod Health Care 2008;34:237-46.

2. Makarainen L, Van Beek A, Tuomivaara L, Asplund B, Bennink HC. Ovarian function during the use of a single contraceptive implant; Implanon compared with Norplant. Fertil Steril 1998; 69:714-21.

3. Timmer CJ, TM Mulders. Pharmacokinetics of etonogestrel and ethinylestradiol released from a combined contraceptive vaginal ring. TMT Clin Pharmacokinet 2000;39:233-42.

4. Timmer CJ, Srivastava N, Dieben TO, Cohen AF. Bioavailability and bioequivalence of etenogestrel from two oral formulations of desogestrel: Cerazette and Liseta. Eur J Metab Pharmacokinet 1999;24:335-43.

5. Thomas T, Petrie K, Shim J, Abildskov KM, Westhoff CL, Cremers S. A UPLC-MS-MS method for therapeutic drug monitoring of etonogestrel. Ther Drug Monit 2013;35:10.

6. Liu XF, Ding CG, Ge QH, Zhou Z, Zhi XJ. Simultaneous determination of gestodene, etonogestrel and ethinylestradiol in plasma by LC-MS/MS following derivatization. Yao Xue Xue Bao 2010;45:87-92.
7. Irving Institute for clinical and translational research. Available from: http://irvinginstitute.columbia.edu/resources/assays/ etonogestrel.html. [Last accessed on 20 Jul 2016]

8. Pena MA, Sanz E, Francisco S, Alonso A, Abajo Z, Felipe I, et al. Randomized, crossover and single-dose bioequivalence study of two oral desogestrel formulations (film-coated tablets of 75 $\mu \mathrm{g}$ ) in healthy female volunteers. Sci Pharm 2012;80:419-31.

9. Moser C, Zoderer D, Luef G, Rauchenzauner M, Wildt L, Griesmacher A, et al. Simultaneous online SPE-LC-MS/MS quantification of six widely used synthetic progestins in human plasma. Anal Bioanal Chem 2012;403:961-72.

10. Bleeker JS, WJ Hogan. Thrombocytosis: diagnostic evaluation, thrombotic Risk stratification, and risk-based management strategies. Thrombosis 2011;1-16. http://dx.doi.org/10.1155/ 2011/536062

11. Castro AD, Concheiro M, Shakleya DM, Huestis MA. Simultaneous quantification of methadone, cocaine, opiates, and metabolites in human placenta by liquid chromatographymass spectrometry. J Anal Toxicol 2009;33:243-52.

12. US DHHS FDA CDER. Guidance for Industry: Bioanalytical Method Validation US Department of Health and Human Services Food and Drug Administration Center for Drug Evaluation and Research CDER Center for Veterinary Medicine CV; 2001.

13. De Boer $\mathrm{T}, \mathrm{J}$ Wieling. Incurred sample accuracy assessment: design of experiments based on standard addition. Bioanalysis 2011;3:983-92.

14. Matta MK, Pilli NR, Inamadugu JK, Burugula L, Rao JVLNS. Simultaneous quantification of lamivudine, zidovudine and nevirapine in human plasma by liquid chromatography-tandem mass spectrometry and its application to a pharmacokinetic study. Acta Pharm Sin B 2012;2:472-80.

15. Matta MK, Pilli NR, Rao JVLNS. A validated liquid chromatography and tandem mass spectrometric method for simultaneous quantitation of tenofovir, emtricitabine and efavirenz in human plasma and its pharmacokinetic application. Acta Chromatographica 2015;27:27-39.

16. Matta MK, Pilli NR, Rao JVLNS. Bioanalysis of raltegravir, an integrase inhibitor in human plasma by novel SPE-ESI-LCMS/MS method and its pharmacokinetic application. Am J PharmaTech Res 2013;3:575-86.

17. Dalia Zidan, Omnia A Ismaiel, Wafaa S Hassan, Abdalla Shalaby. Rapid and validated HPLC-UV method for determination of 
gemifloxacin in human urine. Int $\mathrm{J}$ Pharm Pharm Sci 2015;7:104-8

18. ICH Q2B Validation of Analytical Procedures: Methodology. International Conference on Harmonisation, IFPMA, Geneva; 1996.

19. Athavia BA, Dedania ZR, Dedania RR, Vijayendra Swamy SM, Prajapati CB. Stability indicating HPLC method for determination of vilazodone hydrochloride. Int J Curr Pharm Res 2017;9:123-9.
20. Comparative LC-MS Stability Indicating Assays of Ondansetron Hydrochloride/Naloxone Hydrochloride and Metoclopramide Hydrochloride/Naloxone Hydrochloride Used In Palliative Care; 2015.

21. US Department of Health and Human Services, Food and Drug Administration; 2001. Available from: http:// www.fda.gov/cder/guidance/4252fnl.htm. [Last accessed on 25 Jun 2017] 